\title{
Evidence for spontaneous retrieval of suspended but not finished prospective memories
}

\author{
Michael K. Scullin \\ Washington University, St. Louis, Missouri \\ Gilles O. Einstein \\ Furman University, Greenville, South Carolina \\ AND \\ MARK A. MCDANIEL \\ Washington University, St. Louis, Missouri
}

\begin{abstract}
McDaniel and Einstein (2007) argued that prospective memories can be retrieved through spontaneous retrieval processes stimulated by the presence of a target cue. To test this claim, we investigated whether presenting a prospective memory cue during a task that did not require an intention to be performed spontaneously triggered remembering of that intention. In two experiments, participants performed an image-rating task in which a prospective memory task (to press the "Q" key when a target word appeared) was embedded. Then, participants were told that their intention was finished or suspended. Finally, participants performed a lexical decision task in which each target (and a matched control) word appeared. RTs were slower to target words than to control words when the intention was suspended but not when it was finished. These results suggest that target cues associated with suspended intentions can spontaneously trigger remembering but that finished intentions are quickly deactivated.
\end{abstract}

Prospective memory, or memory for performing intended actions in the future, is critical to everyday functioning. Common prospective memory intentions include remembering to take medication with breakfast and delivering a message to a friend. In the typical laboratory test (Einstein \& McDaniel, 1990) of prospective memory, participants are busily engaged in performing an ongoing task, and on encountering a target event (e.g., a target word), they must disengage the ongoing activity and execute an intended action (i.e., the prospective memory task). Thus, tests of prospective memory are unique (relative to, for example, cued recall and free recall tests of retrospective memory), in that participants must remember to perform the prospective memory task (Craik, 1986).

Two general categories of theories have been developed to explain how one self-initiates retrieval of prospective memory intentions. ${ }^{1}$ Monitoring theories such as the preparatory attentional and memory processes (PAM) theory argue that monitoring or other preparatory processes must be engaged prior to the occurrence of a target event for an intention to be successfully retrieved (Smith, 2003; Smith \& Bayen, 2004; Smith, Hunt, McVay, \& McConnell, 2007). These processes are assumed to be nonautomatic and to require attentional or working memory capacity and may be conscious or unconscious (Smith et al., 2007).
The multiprocess theory (McDaniel \& Einstein, 2000) offers an alternative explanation of prospective memory retrieval. The multiprocess theory argues that in addition to monitoring, intentions may be spontaneously retrieved. The assumption underlying spontaneous retrieval is not that retrieval must be automatic (some facets of retrieval, such as noticing that the cue is related to an intended action, may indeed be automatic, whereas full retrieval may be effortful; Einstein \& McDaniel, 2009) but, instead, that a cue may trigger retrieval even when no attentional resources are being devoted to monitoring for the target cue or maintaining the intention. Thus, in contrast to the PAM theory, the multiprocess theory assumes that, in the absence of monitoring or other resource-consuming processes, prospective memories can be retrieved spontaneously.

One popular approach to conducting prospective memory research and testing the predictions of the PAM and multiprocess theories is to examine task interference (Marsh, Hicks, Cook, Hansen, \& Pallos, 2003). Task interference is observed when adding a prospective memory task reduces performance (speed or accuracy) on an ongoing task. The common assumption is that, if prospective memory retrieval is an effortful process that requires attentional resources (see PAM theory; Smith, 2003) in order to maintain the intention or to monitor for cues, a prospective memory task should negatively affect ongo- 
ing task performance. On the other hand, if prospective memory retrieval is spontaneous and does not require monitoring for cues at the time the target event is encountered (and immediately preceding the target event), high prospective memory performance can be obtained in the absence of task interference (see the multiprocess theory; McDaniel \& Einstein, 2000). The results of research testing for task interference have been mixed (A.-L. Cohen, Jaudus, \& Gollwitzer, 2008; Einstein et al., 2005; Guynn, 2003; Marsh et al., 2003; Smith, 2003; Smith \& Bayen, 2004; Smith et al., 2007), suggesting that the parameters of the ongoing and prospective memory tasks may determine whether prospective memory retrieval requires that attentional resources be allocated to the prospective memory task immediately prior to target events (but see Smith et al., 2007, for an alternative explanation).

Even though the task interference approach has been useful for answering many questions in prospective memory, this approach may not capture certain aspects of prospective memory retrieval in the real world. For example, in the typical task interference paradigm, a prospective memory task is embedded within a specific ongoing activity, and individuals encounter their prospective memory cue within minutes of encoding their intended action. Therefore, this paradigm does not reflect many real-world prospective memory tasks in which there may be substantial delays (hours or days) between encoding and intention execution and the prospective memory cue may appear in an unexpected context (e.g., you may need to remember to deliver a message to a colleague, and it would be useful to retrieve this message if you saw your colleague in any number of situations). The present research was designed to investigate the latter possibility, that prospective memories can be spontaneously retrieved even during contexts in which the prospective memory task is not expected to be performed.

Einstein et al. (2005, Experiment 5) introduced a different procedure for examining spontaneous retrieval of prospective memories. In their paradigm, participants were given two blocks of an ongoing image-rating task in which a prospective memory task (or a retrospective memory control task) was embedded. A lexical decision task, for which participants were told to suspend all other task demands (including the prospective memory demand) and to respond as quickly as possible, was interleaved between the two image-rating blocks. Importantly, the target word and a matched control word were presented once during each lexical decision task, and reaction times (RTs) to these words were recorded. This procedure was repeated 10 times in a prospective memory condition and then another 10 times in a retrospective memory condition (order counterbalanced) with a new target word for each repetition. Einstein et al.'s results demonstrated significant slowing to target words relative to control words during the lexical decision task. Their interpretation was that presentation of the target word spontaneously triggered retrieval of the intention, and this retrieval interfered somewhat with the lexical decision response. This intention interference effect (A.-L. Cohen, Dixon, \& Lindsay, 2005) was unlikely to be explained by devotion of resources to monitoring or rehearsal of the target words, because RTs to the control words in the prospective memory condition did not differ reliably from RTs to the control words in the retrospective memory control condition (i.e., a condition in which monitoring is completely unnecessary and implausible). The findings of no monitoring or cue rehearsal during tasks that do not require a prospective memory task to be performed converge with Marsh, Hicks, and Cook's (2006) results that individuals are able to suspend allocating attention to monitoring for cues or rehearsing the prospective memory intention until the context is one in which the prospective memory task needs to be performed.

Einstein et al.'s (2005) approach to examining spontaneous retrieval seems promising, but their findings may have been unique to their particular procedural features. Specifically, the lexical decision task appeared as blocks of trials that interrupted the ongoing task. Participants had been instructed to perform the prospective memory task during the ongoing image-rating task but to suspend performing it during the lexical decision trials. Therefore, participants were unable to perform their prospective memory task before performing the lexical decision trials, because the prospective memory cue always appeared during the latter half of image-rating trials. This last point is important because there is evidence both that tasks that have not yet been performed (Schiffman \& GreistBousquet, 1992) are kept in a state of greater activation than are performed tasks and that this tendency will lead to continued devotion of attentional resources to the incomplete task. Thus, a possible alternative explanation of Einstein et al.'s procedure was that it represented a Zeigarnik task in which participants were repeatedly forced to delay performance of their prospective memory task. From this perspective, having to repeatedly delay performance of the prospective and retrospective memory tasks may have led some participants to monitor during the lexical decision trials. Furthermore, because the procedure was repeated 10 times in each memory condition with a new target word introduced for each repetition, participants may have been encouraged to occasionally rehearse the target words during the lexical decision task (and in the retrospective memory control blocks) to ensure that they had the appropriate target in mind for that particular block of trials.

The purpose of the present study was to extend Einstein et al.'s (2005, Experiment 5) results, as well as to address these potential limitations. We aimed to more closely implement a typical prospective memory paradigm, in which participants are given one prospective memory task to be performed in the context of a specific ongoing task. Furthermore, we minimized the concern that the procedure may be a Zeigarnik task (as in Einstein et al., 2005, Experiment 5) by having participants perform their prospective memory task several times in a specific context (i.e., an image-rating task). After performing the prospective memory task, participants were instructed that they would need to perform the prospective memory task again at a later point in time (the suspended condition, Experiments 1 and 2) or that the prospective memory task was finished (Experiment 2). Then, participants performed the 
lexical decision task, during which their only goal was to make ongoing task responses as quickly and accurately as possible. During the lexical decision task, we presented the target and matched control words several times and measured RTs to these words. According to the multiprocess theory, presentation of focal target cues can spontaneously trigger remembering, and thus, RTs should be slower to target words than to control words (i.e., intention interference should be obtained).

\section{EXPERIMENT 1}

In Experiment 1, participants initially performed an ongoing image-rating task, along with the prospective memory task of pressing a designated key whenever either of two target words occurred during the image-rating task. Then, participants were instructed to suspend their prospective memory task during several intervening blocks of trials. One block of trials consisted of a lexical decision task, during which participants were told that their sole concern was to perform the task as quickly and accurately as possible. Past research suggests that attentional resources will not be allocated to maintaining or retrieving the prospective memory during the intervals in which the prospective memory task is not to be performed (lexical decision in the present case; see Marsh et al., 2006). ${ }^{2}$ The key feature is that the prospective memory target words (as well as matched control words) were presented several times during the lexical decision task. According to the multiprocess theory's assumption that prospective memory retrieval can be spontaneous when the target event is focally processed (as a word cue should be during a lexical decision task), RTs to the target words should be longer than those to the control words. The idea is that spontaneous retrieval of the prospective memory intention will interfere with the participants' lexical decision processes, thereby slowing responding.

\section{Method}

Participants and Design. The participants were 24 Furman University students receiving credit for a general psychology course. The participants were tested in groups of 1-4. The type of word (target or control) during the lexical decision task was manipulated within subjects.

Procedure. The participants received three successive imagerating phases, followed by a lexical decision task. The participants also performed a prospective memory task during the first imagerating phase. First, the participants received instructions for the image-rating task. They were told to rate the words, as quickly and accurately as possible, on the basis of how easily each word could be mentally imaged (the scale ranged from 1 to 3 , with 1 indicating easy to image and 3 indicating difficult to image). The image-rating instructions were followed by a block of 10 practice trials. Then, the participants received instructions for the prospective memory task. The prospective memory task consisted of remembering to press the "Q" key whenever either of two target words (corn and dancer or fish and writer) occurred in the context of the image-rating task. The two words not used as targets served as control words, matched on frequency and length (Kučera \& Francis, 1967) and were also presented in the lexical decision task. The participants were reminded that their primary goal was to rate words as quickly and accurately as possible. To ensure that the participants understood the tasks, they were required to repeat the instructions to the experimenter.
Then, the participants performed another block of 10 practice trials in which each target word appeared once. After the second imagerating practice block, the participants filled out forms (e.g., a vocabulary test) before completing a block of 80 experimental imagerating trials. During this block, each target word appeared twice, and each control word appeared once (the control words were also presented once in an earlier practice block). Target 1 (i.e., corn or fish) appeared on Trials 15 and 75, whereas Target 2 (i.e., dancer or writer) appeared on Trials 35 and 55. Control 1 appeared on Trial 25, whereas Control 2 appeared on Trial 45.

Following the completion of the first image-rating task, the participants were instructed not to press the "Q" key if they encountered target words for the next few image-rating tasks but to perform the task later in the experiment, after completing the lexical decision task. Thus, the participants were told to suspend their prospective memory task for several blocks. Then, the participants received a third block of 10 image-rating practice trials, followed by a request to fill out more forms. Once completed, the participants performed a block of 80 experimental image-rating trials (Control 1 appeared on Trial 45 and Control 2 appeared on Trial 25) followed by a short block of 24 image-rating trials. No target words appeared during these blocks.

Next, the participants were given the instructions for the lexical decision task (referred to as the speed task). The participants were instructed to decide whether a string of letters formed a word as quickly and accurately as possible by pressing the keys labeled " $Y$ " or " $\mathrm{N}$ " (the " 5 " and " 6 " keys on the number pad, respectively). To ensure that they understood the instructions, the participants were required to explain the lexical decision task to the experimenter. After explaining the instructions, the participants completed a block of 22 lexical decision practice trials in which they received feedback based on their speed and accuracy. After the practice block, the participants performed 280 lexical decision trials. Each target and control word was presented five times. Target 1 was presented on Trials $55,94,165,214$, and 263, and Target 2 was presented on Trials 32, $98,128,187$, and 241. Control 1 was presented on Trials 23, 88, 172, 217, and 250, and Control 2 was presented on Trials 44, 123, 136, 196 , and 254. The target and control words were counterbalanced, so that one group's target words were another group's control words.

Materials. All participants were tested on Compaq computers using an E-Prime program in Windows. Image-rating and lexical decision items were obtained from the Kučera and Francis (1967) word norms and were of medium frequency. Nonwords were created from words by switching one or two consonants, with the stipulation that the nonword remained pronounceable.

\section{Results and Discussion}

For all analyses reported throughout this study, we set $\alpha=.05$ for inferring statistical significance.

Prospective memory. Although not of central interest in this experiment, the proportion of prospective memory trials on which participants remembered to press the "Q" key during the image-rating task was measured. Prospective memory performance was high $(M=.91)$.

Lexical decision performance. Consistent with previous research (Einstein et al., 2005), we trimmed RTs for each participant to include only correct responses that were fewer than two standard deviations away from the mean. The trimming method resulted in the elimination of $5.0 \%$ of RTs. A within-subjects ANOVA was conducted, comparing the trimmed mean RTs of target and control words. The participants responded significantly more slowly to target words $(M=533 \mathrm{msec})$ than to control words $(M=512 \mathrm{msec})\left[F(1,23)=4.53, M S_{\mathrm{e}}=1,147.99\right.$, $p=.04] .{ }^{3}$ This result suggests that on target trials, some aspect of the prospective memory intention was retrieved 
or noticed, thereby interfering with the lexical decision response.

Furthermore, it is highly likely that such retrieval (or noticing) was spontaneous (see A.-L. Cohen et al., 2005, for a similar interpretation). The lexical decision task occurred after performance of the prospective memory task (see the Zeigarnik task for comparison: Schiffman \& Greist-Bousquet, 1992), thereby attenuating possible confusion for the participants about when the prospective memory task should be performed. By contrast, in the Einstein et al. (2005, Experiment 5) paradigm, because the lexical decision task interrupted the image-rating task and occurred before prospective memory trials, it is possible that their participants were occasionally confused and monitored. In the present experiment, the participants were highly familiar with the ongoing activity in which the prospective memory task was embedded, because they were required to explain the instructions to the experimenter, and they performed their prospective memory task twice during a practice image-rating block and four times during the experimental image-rating block (mean prospective memory performance $=.91$ ). Therefore, the participants should not have been confused about whether the lexical decision task also included a prospective memory task. Under such conditions, the participants typically do not monitor for the target in the phase in which the prospective memory task is not expected (see Marsh et al., 2006, for strong evidence on the point).

\section{EXPERIMENT 2}

In Experiment 1, we established that target cues associated with suspended prospective memory tasks were noticed or otherwise triggered spontaneous retrieval processes during an ongoing task context that did not require the prospective memory task to be performed. An interesting follow-up question concerns whether these spontaneous retrieval processes can be turned off by instructing participants that their prospective memory task is finished. Whether spontaneous retrieval processes can be deactivated is an important question, because prospective memory retrieval has been demonstrated during ongoing task contexts that have no embedded prospective memory task (Experiment 1), which implies that one retrieves intentions even when it is unnecessary to do so (see Kvavilashvili \& Fisher, 2007, for a similar finding in a naturalistic study). However, it would be maladaptive if irrelevant (i.e., finished) intentions constantly popped into mind whenever an old cue appeared. For example, after remembering to return a book to the library, it would be adaptive to not continually retrieve the old intention to return the book when walking past the library. Consistent with this view, in an experiment in which participants were required to learn to-be-performed scripts (e.g., brewing coffee), after script completion, intentions "appear[ed] to undergo deactivation, or inhibition" (Marsh, Hicks, \& Bink, 1998, p. 356). Because spontaneous retrieval appears to occur (Einstein et al., 2005; Mace, 2007; Scullin, McDaniel, \& Einstein, 2008), and because people forget old intentions (Shapiro \&
Krishnan, 1999), a plausible assumption is that spontaneous retrieval processes are deactivated following prospective memory task completion.

In Experiment 2, after the participants completed the experimental image-rating phase in which they performed their prospective memory task, we told them that their prospective memory task would be performed again later (i.e., the suspended condition) or that their prospective memory task was finished (the finished condition). The finished condition not only allowed us to examine whether spontaneous retrieval processes were deactivated, it also removed any reason for the participants to continue monitoring (if they had been to begin with). Therefore, the lexical decision RTs on control trials in the finished condition provided us with a no-monitoring baseline with which to compare the control trials in the suspended condition. If the RTs to control trials in the suspended condition are equivalent to those in the finished condition, one can conclude that the participants were not monitoring (in the suspended condition).

One final aspect of Experiment 2 deserves mention. Instead of presenting the prospective memory task only during the first image-rating phase (Experiment 1), we manipulated whether the participants performed the task during the first image-rating phase (which had a long delay between the prospective memory task and the lexical decision task) or the second image-rating phase (the short-delay condition). By manipulating delay, we were able to test whether the intention interference effect in the suspended condition (i.e., slowed responding on target trials) and the hypothesized deactivation of intentions in the finished condition depend on the delay between performing the prospective memory task and processing the target words during the lexical decision task.

\section{Method}

Participants and Design. The participants were 96 Furman University students receiving credit for a general psychology course. The experiment featured a $2 \times 2 \times 2$ mixed factorial design that included prospective memory task instructions (suspended or finished) and delay (short or long) between performing the prospective memory and lexical decision tasks as between-subjects factors and type of word (target or control) in the lexical decision task as a within-subjects factor.

Procedure. The procedure was identical to that in Experiment 1, with the following exceptions. First, the image-rating phase in which participants received the prospective memory task instructions varied. In the long-delay condition, the participants received their prospective memory task during the first image-rating phase. In the short-delay condition, the participants performed the prospective memory task during the second image-rating phase. Target events occurred during practice and experimental trials only during the image-rating phase in which participants received the prospective memory task instructions (i.e., the first or the second image-rating phase). Immediately following completion of the image-rating phase in which the prospective memory task was performed, the participants received instructions that their prospective memory task would be performed again (i.e., the suspended condition; the instructions were identical to those in Experiment 1) or that it was finished (i.e., the finished condition). In the finished control condition, the participants were told that they no longer needed to press " $Q$ " for their target words.

Materials. All participants were tested on Compaq computers using an E-Prime program in Windows. Image-rating, lexical 
decision, target, and control items were identical to those used in Experiment 1

\section{Results and Discussion}

Prospective memory performance. Performance on the prospective memory task was calculated as the proportion of times in which the "Q" key was pressed during or immediately following target trials. We conducted a $2 \times 2$ ANOVA that included delay (short or long) and prospective memory instructions (suspended or finished) as between-subjects factors. There was no effect of delay or prospective memory instructions (both $F \mathrm{~s}<1$ ) on prospective memory performance, but the interaction was significant $\left[F(1,96)=3.96, M S_{\mathrm{e}}=.07, p=.05\right]$. In the short-delay condition, prospective memory was higher in the finished condition $(M=.97)$ than in the suspended condition $(M=.84)$, whereas, in the long-delay condition, prospective memory was greater in the suspended condition $(M=.91)$ than in the finished condition $(M=$ .82). There is no apparent substantive interpretation of this pattern, because the manipulation of prospective memory instructions followed performance of the prospective memory task. Most likely, especially because overall prospective memory performance was near ceiling and variability was therefore artificially reduced, this interaction represents a Type I error.

Lexical decision performance. Consistent with Experiment 1 , we examined trimmed (i.e., correct trials within two standard deviations of mean) RTs on target and control trials during the lexical decision task. Using the dependent measure of trimmed RTs, we conducted a $2 \times 2 \times 2$ mixed ANOVA that included delay (short or long) and prospective memory instructions (suspended or finished) as between-subjects factors and type of word in the lexical decision task (target or control) as a withinsubjects factor. The trimming method resulted in elimination of approximately $5.3 \%$ of responses, and the only significant effect was the interaction between the type of word in the lexical decision task and prospective memory instructions $\left[F(1,96)=3.91, M S_{\mathrm{e}}=2,019.39, p=.05\right]$ (see Figure 1). Planned comparisons confirmed that RTs were significantly longer on target trials $(M=558 \mathrm{msec})$ than on control trials $(M=539 \mathrm{msec})$ in the suspended condition $\left[F(1,96)=4.21, M S_{\mathrm{e}}=2,019.39\right]$, but in the finished condition, RTs were similar $(F<1)$ for target trials $(M=532 \mathrm{msec})$ and control trials $(M=539 \mathrm{msec}){ }^{4}$

Can the intention interference effect in the suspended condition be attributed to engaging monitoring or rehearsal processes during the lexical decision task? To address this question, we conducted a planned comparison of RTs on control trials between the suspended condition and the finished condition (i.e., the condition in which participants would not be monitoring). The planned comparison revealed no difference between conditions in responding to control words (both $M \mathrm{~s}=539 \mathrm{msec}, F<1$; see Figure 1). Thus, the retrieval demonstrated in the suspended condition cannot be explained by participants engaging monitoring or rehearsal processes. Instead, presentation of the target during the lexical decision task appears to have spontaneously reminded participants of their prospective memory task, and this process slowed lexical decision responding.

One potential concern over finding the intention interference effect in Experiments 1 and 2 was that presenting the control words during the image-rating task may have resulted in repetition priming. If repetition priming occurred for control words (and not for target words), the intention interference effect may have been artificially augmented. To investigate this issue, we tested an additional 54 participants in the finished condition (short or long delay) and included an additional set of control words (e.g., moon and artist) that were never presented during the image-rating task. Thus, during the lexical decision task, two target words, two previously presented control words, and two never-before-presented control words each

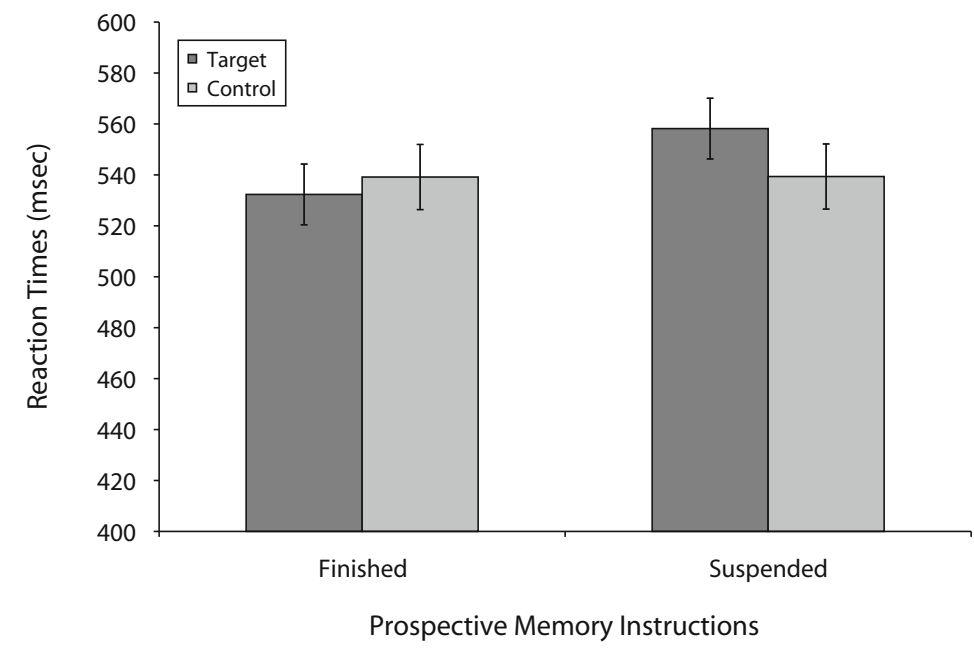

Figure 1. Mean trimmed lexical decision reaction times to target and control words across the finished and suspended conditions in Experiment 2. 
appeared five times. Each pair of words (i.e., corn/dancer, fish/writer, or moon/artist) appeared an equal number of times as target words, previously presented control words, and never-before-presented control words.

Using the dependent measure of trimmed RTs during the lexical decision task, we conducted a $3 \times 2$ mixed ANOVA in which type of word during the lexical decision task (target, previously presented control, never-beforepresented control) varied within subjects and delay (short or long) varied between subjects. There were no significant effects [largest $F(1,52)=2.18, M S_{\mathrm{e}}=1,036.86$, for the delay $\times$ type of word interaction]; following completion of the prospective memory task, responding to target words $(M=525 \mathrm{msec})$ did not differ from responding to previously presented $(M=518 \mathrm{msec})$ or never-beforepresented $(M=513 \mathrm{msec})$ control words. Therefore, we were able to replicate the null effect of no intention interference after the prospective memory task had been finished. Furthermore, a planned comparison showed no significant difference $(F<1)$ in RTs to previously presented and never-before-presented control words, thereby demonstrating that the intention interference effect cannot be explained by repetition priming.

\section{GENERAL DISCUSSION}

The results of the present research replicate Einstein et al.'s (2005) results by demonstrating longer RTs to target words than to control words when the prospective memory task was suspended (i.e., the intention interference effect; A.-L. Cohen et al., 2005). This effect occurred during a phase that did not require monitoring processes to be engaged or the prospective memory task to be performed. In this section, we address how the intention interference effect can inform theories of prospective memory retrieval, why the effect is eliminated if the prospective memory phase is finished, and whether the intention interference effect represents a familiarity-based process (e.g., noticing) or intention retrieval.

The first question that we wish to address is how the intention interference effect could inform the debate over the mechanism(s) underlying prospective memory retrieval. The PAM theory (Smith, 2003; Smith \& Bayen, 2004; Smith et al., 2007) argues that, during the interval in which the prospective memory task must be executed, "successful true [prospective memory] performance requires that preparatory attentional processes be engaged" (Smith et al., 2007, p. 742). Furthermore, the PAM theory argues that we do not have to be consciously aware of the engagement of preparatory (e.g., monitoring) processes, but nevertheless, these processes will consume attentional resources. In the present research (Experiment 2), however, there was no evidence for decreased availability of attentional resources in the suspended condition, because lexical decision control trial RTs were identical in the suspended and finished conditions. Despite an absence of preparatory processes during the lexical decision task in the suspended condition, the prospective memory target words triggered some additional processing that may represent retrieval. Although the PAM theory focuses on retrieval during the performance interval, whereas the present experiments examined retrieval outside of the designated performance interval, the finding that some aspect of the prospective memory intention may be retrieved in the absence of preparatory processes does not conform to the current specifications of the PAM theory.

In contrast to the PAM theory, the multiprocess theory (Einstein et al., 2005; McDaniel \& Einstein, 2007) argues that, in the absence of preparatory (e.g., monitoring) processes, individuals may spontaneously retrieve their intentions. According to the multiprocess theory, spontaneous retrieval of the intended action is likely when participants have formed a good encoding of the target cue and a good association between the target cue and the intended action. Also important is that the ongoing task encourages focal processing of the target cue. By this, we mean that the ongoing task directs attention to the target cue and to those features that were processed at encoding (see Einstein \& McDaniel, 2005, and Breneiser, McDaniel, \& Derbish, 2008, for examples of focal and nonfocal processing). This thinking follows directly from the encoding specificity principle (Tulving, 1983): Features of the cue that are processed at retrieval need to match those features that were processed at encoding for successful retrieval to occur. We used focal cues (i.e., the target words were focally processed, because the lexical decision task directs attention toward processing whole words) in the present research and, thus, conditions that, according to the multiprocess theory (McDaniel \& Einstein, 2007), encourage spontaneous retrieval.

The present finding that participants responded more slowly to prospective memory target words than to control words (in the suspended conditions) supports the multiprocess theory's (McDaniel \& Einstein, 2007) prediction that, in the absence of monitoring or other preparatory processes, focal cues associated with a suspended prospective memory task can spontaneously trigger retrieval of the intention. Thus, the present research converges with those studies examining task interference showing that, in the absence of monitoring (i.e., nonsignificant task interference), individuals may spontaneously retrieve their prospective memory intentions (A.-L. Cohen et al., 2008; Einstein et al., 2005, Experiments 1-4; Scullin et al., 2008). Notably, our paradigm for investigating spontaneous retrieval may have some advantages over the more traditional task interference approach. First, examining RTs on target trials during a task that does not require execution of the prospective memory action is a more precise measure than is the task interference approach. Because monitoring and/or rehearsal of the prospective memory intention (inferred from task interference) presumably waxes and wanes across ongoing task blocks (see, e.g., West \& Craik, 1999) and task interference is usually averaged across entire blocks, it is difficult to use task interference to determine whether prospective memory retrieval on any given trial was spontaneous or required monitoring (or other preparatory) processes. In contrast, the present approach allows a more direct examination of the process of retrieval because it tests for changes in processing (i.e., slowing) to the target items themselves. Thus, in addition 
to the task interference approach, the present methodology is an informative means to study spontaneous retrieval.

Interestingly, the intention interference effect was not demonstrated in all conditions. Specifically, there was evidence for intention interference (i.e., spontaneous retrieval) when the prospective memory task was suspended but not when it was finished. This finding supports the hypothesis that finished prospective memory intentions undergo deactivation (Marsh et al., 1998). The hypothesis that finished intentions are quickly deactivated stems from research reporting the intention superiority effect (Goschke \& Kuhl, 1993). In this paradigm, participants typically learn two scripts (e.g., the steps for setting a table), one that they are instructed they will perform (or, in a control condition, observe the experimenter perform) and one that they will not perform (the neutral script). Then, prior to performing the script, participants are given a recognition task in which they must decide as quickly as possible whether an action is from the perform script. The intention superiority effect is demonstrated when participants recognize items from the perform script faster than those from the neutral script when the intention is going to be performed (but not when it is only to be observed), implying that intentions are stored at a higher level of activation than are neutral items.

Pertinent for the present purposes, to assess activation, Marsh et al. (1998) gave participants a lexical decision task (instead of a recognition test) containing nouns and verbs from the scripts after the participants performed (or observed) the actions from the perform script. Consistent with Goschke and Kuhl's (1993) results, there was no difference in RTs to neutral and to script items when the intention was observed. After performing a script, however, the participants responded more slowly to items from that script than to neutral script items. Marsh et al.'s (1998) finding of slower responding to performed items than to neutral items after the intention (i.e., the script) was performed demonstrates a change in activation of the intention (relative to the higher activation associated with the intention superiority effect). Although Marsh et al. (1998) used an intention superiority effect paradigm and not a standard prospective memory task, their results provided initial evidence that, once a prospective memory task is finished, it is inhibited or otherwise deactivated.

Instead of focusing on the activation of script items, we tested for the existence of spontaneous retrieval of the prospective memory intention when the target cue was focally processed, and we tested this before and after completion of the prospective memory task. Interestingly, the intention interference effect was demonstrated when the prospective memory task was suspended but not when it was finished. Thus, this pattern is consistent with Marsh et al.'s (1998) intention superiority results and offers further evidence that our cognitive system deactivates finished intentions. Taken together, these findings are consistent with the intuitive notion that intentions are forgotten (Shapiro \& Krishnan, 1999) and suggest a highly adaptive function of this type of forgetting. Because individuals form and complete many intentions every day, an inability to forget these finished intentions would interfere with daily functioning.

A possible mechanism responsible for the flexibility of spontaneous retrieval processes lies in the functioning of the hippocampus. Many theorists (e.g., J. D. Cohen \& O'Reilly, 1996; Eichenbaum, Yonelinas, \& Ranganath, 2007; Moscovitch, 1994) suggest that the hippocampus functions as an associative memory mechanism that binds contiguously present information (such as an anticipated cue and an intended action). Then, when part of that bound information is subsequently encountered (i.e., the cue), provided that the association is strong enough and that the encountered information is fully processed, this hippocampal mechanism reflexively delivers to awareness the remainder of the information (i.e., the intended action that is associated with the encountered cue; J. D. Cohen \& O’Reilly, 1996; Moscovitch, 1994). Therefore, the intention interference effect in the suspended condition may reflect additional hippocampal processing of target cues or retrieval of the associated action. More speculatively, in addition to quickly forming associations between items, the hippocampus might function to rapidly disassemble such associations when they are no longer functional (or need to be corrected, as in supervised learning situations). The idea here is that, following prospective memory task completion, a rapid associative memory mechanism (hippocampus) could disassemble or deactivate the association between the target cue and the intended action. At this point, the target cue would no longer stimulate spontaneous retrieval of the prospective memory intention, consistent with the absence of intention interference in the finished condition (Experiment 2).

West, McNerney, and Travers (2007) also investigated the fate of prospective memories following instructions to forget cues. In their study, participants received a different prospective memory cue before each block of a semantic judgment task and were instructed to either perform the prospective memory task or to forget about it for that block. Their results demonstrated longer RTs to the prospective memory cue than to a control word during both prospective perform and prospective forget blocks. Three potentially important differences between the present research and West et al.'s study may explain why they found slowing to prospective memory cues in a forget condition and we did not. First, the delay between cue cancellation and cue presentation was much shorter in West et al.'s study. Second, in the present study, the prospective memory task was performed in the image-rating phase, but the cue was reencountered in the lexical decision phase. In West et al.'s study, the prospective memory cue appeared in the same context in which it would have otherwise been performed (i.e., the semantic judgment task). Finally, participants in the present study performed the prospective memory task before being instructed that it was finished; participants in West et al.'s study never performed the prospective memory action to the cue that they were told to forget (see Zeigarnik tasks; Schiffman \& Greist-Bousquet, 1992). Therefore, whether prospective memories are quickly deactivated may not only depend 
on temporal and contextual factors but also on whether the prospective memory task was performed.

One final question concerns which aspect of the target cue or retrieved prospective memory intention caused participants in the suspended conditions to respond more slowly to target words than to control words during the lexical decision task. There are two hypotheses regarding the spontaneous retrieval mechanism that may inform this slowing. One possibility is that the target cue reflexively triggers retrieval of components of the intended action. Another possibility is that presentation of the target cue leads to discrepant processing, which leads to some noticing of the significance of the cue, which in turn slows the lexical decision (see McDaniel, Guynn, Einstein, \& Breneiser, 2004). The latter possibility fits well with Marsh, Hicks, and Watson's (2002) proposed microstructure of prospective memory retrieval and execution. This microstructure includes cognitive processes relevant to noticing the prospective memory cue, retrieving the intention, and coordinating intention execution. Therefore, in the present experiment, the intention interference effect may have been caused by automatic noticing of the prospective memory cue (perhaps because of discrepant processing) during the lexical decision task or by some combination of noticing and retrieval. If the intention interference effect is a result of both noticing and retrieval processes, one might expect to find slower responding on both target trials and trials following targets (relative to control and control +1 trials). Although the present research was not designed to investigate this issue (lexical decision items following target trials differed from those following control trials), our lab has collected some unpublished data that demonstrate that intention interference continues on target +1 trials (relative to control +1 trials) before dissipating over the subsequent two trials. Therefore, intention interference may be caused by spontaneous noticing on target events followed by retrieval of the prospective memory intention (evidenced by slowing on the subsequent trial).

In conclusion, the present research raises the interesting issue of how much information is retrieved when a prospective memory target cue is encountered. The present research suggests that an important variable is whether the prospective memory task is suspended (i.e., activated) or finished (i.e., deactivated). Interesting avenues for future research may involve examining the range of contexts in which prospective memory target cues trigger retrieval processes, as well as the mechanisms that underlie activation and deactivation of these retrieval processes. In addition to informing theories of prospective memory retrieval, such research will help us understand how individuals perform daily activities efficiently in a world in which intentions are constantly formed, completed, and forgotten.

\section{AUTHOR NOTE}

Portions of this project were presented at the 48th Annual Meeting of the Psychonomic Society, Long Beach, CA, and as a thesis at Furman University. Correspondence concerning this article should be addressed to M. K. Scullin, Department of Psychology, Washington University, St. Louis, MO 63130-4899 (e-mail: mscullin@wustl.edu).

\section{REFERENCES}

Breneiser, J. E., McDaniel, M. A., \& Derbish, M. (2008). Prospective memory targets: Focal and task-appropriate. Manuscript submitted for publication.

Cohen, A.-L., Dixon, R. A., \& Lindsay, D. S. (2005). The intention interference effect and aging: Similar magnitude of effects for young and old adults. Applied Cognitive Psychology, 19, 1177-1197.

Cohen, A.-L., Jaudus, A., \& Gollwitzer, P. M. (2008). Number of cues influences the cost of remembering to remember. Memory \& Cognition, 36, 149-156.

Cohen, J. D., \& O'Reilly, R. C. (1996). A preliminary theory of the interactions between prefrontal cortex and hippocampus that contribute to planning and prospective memory. In M. Brandimonte, G. O. Einstein, \& M. A. McDaniel (Eds.), Prospective memory: Theory and applications (pp. 267-295). Mahwah: NJ: Erlbaum.

CraIK, F. I. M. (1986). A functional account of age differences in memory. In F. Klix \& H. Hagendorf (Eds.), Human memory and cognitive capabilities: Mechanisms and performances (pp. 409-422). Amsterdam: North-Holland.

Eichenbaum, H., Yonelinas, A. P., \& Ranganath, C. (2007). The medial temporal lobe and recognition memory. Annual Review of Neuroscience, 30, 123-152.

Einstein, G. O., \& McDaniel, M. A. (1990). Normal aging and prospective memory. Journal of Experimental Psychology: Learning, Memory, \& Cognition, 16, 717-726.

Einstein, G. O., \& McDaniel, M. A. (2005). Prospective memory: Multiple retrieval processes. Current Directions in Psychological Science, 14, 286-290.

Einstein, G. O., \& McDaniel, M. A. (2009). Prospective memory and what costs do not reveal about retrieval processes: A commentary on Smith, Hunt, McVay, and McConnell (2007). Manuscript submitted for publication.

Einstein, G. O., McDaniel, M. A., Thomas, R., Mayfield, S., Shank, H., Morrisette, N., \& Breneiser, J. (2005). Multiple processes in prospective memory retrieval: Factors determining monitoring versus spontaneous retrieval. Journal of Experimental Psychology: General, 134, 327-342.

Goschke, T., \& Kunl, J. (1993). Representation of intentions: Persisting activation in memory. Journal of Experimental Psychology: Learning, Memory, \& Cognition, 19, 1211-1226.

GuYNN, M. J. (2003). A two-process model of strategic monitoring in event-based prospective memory: Activation/retrieval mode and checking. International Journal of Psychology, 38, 245-256.

KuČera, H., \& Francis, W. N. (1967). Computational analysis of present-day American English. Providence, RI: Brown University Press.

KvavilashviLI, L., \& Fisher, L. (2007). Is time-based prospective remembering mediated by self-initiated rehearsals? Role of incidental cues, ongoing activity, age, and motivation. Journal of Experimental Psychology: General, 136, 112-132.

Mace, J. H. (ED.) (2007). Involuntary memory. Malden, MA: Blackwell.

Marsh, R. L., Hicks, J. L., \& Bink, M. L. (1998). Activation of completed, uncompleted, and partially completed intentions. Journal of Experimental Psychology: Learning, Memory, \& Cognition, 24, 350361.

Marsh, R. L., Hicks, J. L., \& CooK, G. I. (2006). Task interference from prospective memories covaries with contextual associations of fulfilling them. Memory \& Cognition, 34, 1037-1045.

Marsh, R. L., Hicks, J. L., Cook, G. I., Hansen, J. S., \& Pallos, A. L. (2003). Interference to ongoing activities covaries with the characteristics of an event-based intention. Journal of Experimental Psychology: Learning, Memory, \& Cognition, 29, 861-870.

Marsh, R. L., Hicks, J. L., \& Watson, V. (2002). The dynamics of intention retrieval and coordination of action in event-based prospective memory. Journal of Experimental Psychology: Learning, Memory, \& Cognition, 28, 652-659.

McDaniel, M. A., \& Einstein, G. O. (2000). Strategic and automatic processes in prospective memory retrieval: A multiprocess framework. Applied Cognitive Psychology, 14, S127-S144.

McDaniel, M. A., \& Einstein, G. O. (2007). Prospective memory: An 
overview and synthesis of an emerging field. Thousand Oaks, CA: Sage.

McDaniel, M. A., Guynn, M. J., Einstein, G. O., \& Breneiser, J. (2004). Cue-focused and reflexive-associative processes in prospective memory retrieval. Journal of Experimental Psychology: Learning, Memory, \& Cognition, 30, 605-614.

Moscovitch, M. (1994). Memory and working with memory: Evaluation of a component process model and comparisons with other models. In D. L. Schacter \& E. Tulving (Eds.), Memory systems (pp. 269310). Cambridge, MA: MIT Press.

Schiffman, N., \& Greist-Bousquet, S. (1992). The effect of task interruption and closure on perceived duration. Bulletin of the Psychonomic Society, 30, 9-11.

Scullin, M. K., McDaniel, M. A., \& Einstein, G. O. (2008, November). Control of monitoring in prospective memory: Evidence for a multiprocess theory. Poster presented at the 49th Annual Meeting of the Psychonomic Society, Chicago, IL.

Shapiro, S., \& Krishnan, H. S. (1999). Consumer memory for intentions: A prospective memory perspective. Journal of Experimental Psychology: Applied, 5, 169-189.

Sмiтh, R. E. (2003). The cost of remembering to remember in eventbased prospective memory: Investigating the capacity demands of delayed intention performance. Journal of Experimental Psychology: Learning, Memory, \& Cognition, 29, 347-361.

SMith, R. E., \& BAYEN, U. J. (2004). A multinomial model of eventbased prospective memory. Journal of Experimental Psychology: Learning, Memory, \& Cognition, 30, 756-777.

Smith, R. E., Hunt, R. R., McVay, J. C., \& McConnell, M. D. (2007). The cost of event-based prospective memory: Salient target events. Journal of Experimental Psychology: Learning, Memory, \& Cognition, 33, 734-746.

Tulving, E. (1983). Elements of episodic memory. Oxford: Oxford University Press.

West, R., \& CraIK, F. I. M. (1999). Age-related decline in prospective memory: The roles of cue accessibility and cue sensitivity. Psychology \& Aging, 14, 264-272.

West, R., McNerney, M. W., \& Travers, S. (2007). Gone but not forgotten: The effects of cancelled intentions on the neural correlates of prospective memory. International Journal of Psychophysiology, 64, 215-225.

\section{NOTES}

1. Throughout this article, we use the terms prospective memory intention and prospective memory retrieval in a theoretically neutral sense. We are not arguing that these constructs are necessarily unique to prospective memory or that they are qualitatively different from the processes supporting retrospective memory. Instead, we use these terms only to refer to the particular memory task (i.e., prospective memory) being used.

2. Conditions in which the prospective memory task has been suspended have been previously used as control groups for which to demonstrate monitoring (Smith, 2003).

3. To ensure that this finding was not a result of the trimming method, we conducted another within-subjects ANOVA to compare RTs on all correct target and control trials (only $1.0 \%$ of trials excluded). The results again demonstrated significantly longer RTs on target trials $(M=$ $544 \mathrm{msec})$ than on control trials $(M=519 \mathrm{msec})\left[F(1,23)=6.38, M S_{\mathrm{e}}=\right.$ $1,245.15, p=.02]$.

4. We also checked that the same pattern holds when examining correct untrimmed RTs (only $1.7 \%$ of responses were excluded). A $2 \times 2 \times 2$ mixed ANOVA on these data that included the between-subjects factors of prospective memory instructions (suspended or finished) and delay (short or long) and the within-subjects factor of type of word during the lexical decision task (target or control) paralleled the results of the trimmed analyses. Again, the only significant effect was the interaction between the type of lexical decision word and prospective memory instructions $[F(1,96)=$ 4.70, $\left.M S_{\mathrm{e}}=2,534.89, p=.03\right]$. Planned comparisons confirmed that RTs were significantly longer on target trials $(M=581 \mathrm{msec})$ than on control trials $(M=558 \mathrm{msec})$ in the suspended condition $\left[F(1,96)=4.65, M S_{\mathrm{e}}=\right.$ 2,534.89]; however, RTs to target $(M=542 \mathrm{msec})$ and to control $(M=$ $551 \mathrm{msec}$ ) words did not differ in the finished condition $(F<1)$. Furthermore, there was no significant difference in control trial RTs between the suspended and the finished conditions $(F<1)$.

(Manuscript submitted July 16, 2008; revision accepted for publication December 26, 2008.) 